\title{
High energy neutrinos to see inside the Earth
}

\author{
E. Borriello ${ }^{1,2}$, G. De Lellis ${ }^{1,2}$, G. Mangano ${ }^{2,1}$, A. Marotta ${ }^{1,2}$, G. Miele $^{1,2,3}$, \\ P. Migliozzi ${ }^{2,1}$, C. Moura' ${ }^{2}$, S. Pastor ${ }^{3}$, O. Pisanti ${ }^{1,2}$, \\ P. Strolin ${ }^{1,2}$, and A. Zollo ${ }^{1}$ \\ ${ }^{1}$ Università “Federico II”, Dipartimento di Scienze Fisiche, Complesso Universitario di Monte S.Angelo, \\ Via Cithia, 80126, Napoli, Italy \\ ${ }^{2}$ INFN_Sezione di Napoli-Complesso Universitario di Monte S.Angelo, Via Cithia, 80126, Napoli, Italy \\ ${ }^{3}$ Instituto de Física Corpuscular (CSIC-Universitat de València), Ed. Institutos de Investigación, \\ Apartado de Correos 22085, E-46071 València, Spain
}

(Received October 29, 2008; Revised June 17, 2009; Accepted June 17, 2009; Online published February 22, 2010)

\begin{abstract}
The new chances offered by elementary particles as probes of the internal structure of our planet are briefly reviewed, by paying particular attention to the case of high energy neutrinos. In particular, the new results concerning the shadow of mountains on $v_{\tau}$ flux at Pierre Auger Observatory is briefly discussed, and moreover the possibility to use the tail of atmospheric neutrinos to probe the core/mantle transition region is just sketched.

Key words: High energy cosmic rays, neutrinos, Earth radial density profile.
\end{abstract}

\section{Introduction}

The exploration of the internal structure of the Earth by using elementary particles is what can be now denoted as Geoparticle Physics. The idea is quite old and started with the determination of the thickness of snow layers on a mountain by means of atmospheric muons (George, 1955). The first application of this method was realized to search for unknown burial cavities in the Chephren's pyramid (Alvarez et al., 1970), but only in recent times it has entered in a phase of first production of quantitative estimates and data, with a general renewed vitality which makes these times very attractive.

This new discipline can be split in two main research fields essentially defined by the nature of the probe: cosmic radiation at high energy (muons or neutrinos) or low energy neutrinos produced by radioactive decays inside the Earth (geo-neutrinos). Hereafter we will focus our attention on the high energy radiation and in particular on the possibility to use neutrinos to probe the very inner part of our planet.

High Energy (HE) neutrino detection is one of the most promising research lines in astroparticle physics. Neutrinos are in fact one of the main components of the cosmic radiation in the high energy regime, and although their fluxes are uncertain and depend on the specific production process, their detection would provide valuable information concerning the sources and the acceleration mechanism in extreme astrophysical environments. For this reason the experimental community is undertaking a relevant effort to construct giant Neutrino Telescopes (NT's). From this point of view, after the first generation of telescopes has proved the viability of the Cerenkov detection tech-

Copyright (c) The Society of Geomagnetism and Earth, Planetary and Space Sciences (SGEPSS); The Seismological Society of Japan; The Volcanological Society of Japan; The Geodetic Society of Japan; The Japanese Society for Planetary Sciences; TERRAPUB.

doi:10.5047/eps.2009.06.002 nique under deep water (Balkanov et al., 1999) and ice (Ahrens et al., 2002) by detecting atmospheric neutrinos, one is probably on the verge of the first detections at the IceCube (Ahrens et al., 2004) telescope, being completed at the South Pole, and possibly at the smaller ANTARES (Spurio, 2006) telescope under construction in the Mediterranean. Additionally, ANTARES as well as NESTOR (Aggouras et al., 2006) and NEMO (Migneco et al., 2008) are involved in $R \& D$ projects aimed at the construction of a $\mathrm{km}^{3} \mathrm{NT}$ in the deep water of the Mediterranean sea, coordinated in the European network KM3NeT (Katz, 2006). In this very exciting scientific framework it has been proposed the idea to use neutrinos, which are elusive particles, to probe the very internal part of the Earth.

In order to use HE neutrinos for geological purposes one has two possible choices: either using an almost known neutrino flux, like in the case of atmospheric neutrinos, or to use the unknown, but expected isotropic and more energetic, extragalactic flux (see Fig. 1). In these two cases the neutrino energies involved are very different and thus consequently the lengths probed (see Fig. 2). The less energetic atmospheric neutrinos, since they have a larger interaction length and can cross distances in rock of the order or larger than the Earth radius, can be used to study the mantle/core structure of our planet. On the other side, UHE neutrinos, like the extragalactic component, may only cross much shorter distances and thus can be used to probe superficial structures (Earth-skimming particles).

\section{Neutrino Sensitivity to Matter Distribution}

In order to understand how the number of charged lepton events at a km $\mathrm{km}^{3} \mathrm{NT}$ depends on the density of matter crossed by HE neutrinos, let us remind that the events rate can be written, neglecting for simplicity the energy dependence, as

$$
\Gamma\left(E_{v}\right)=\int_{\Omega_{\mathrm{d}}} d \Omega A_{\mathrm{eff}} \frac{d \Phi}{d \Omega},
$$




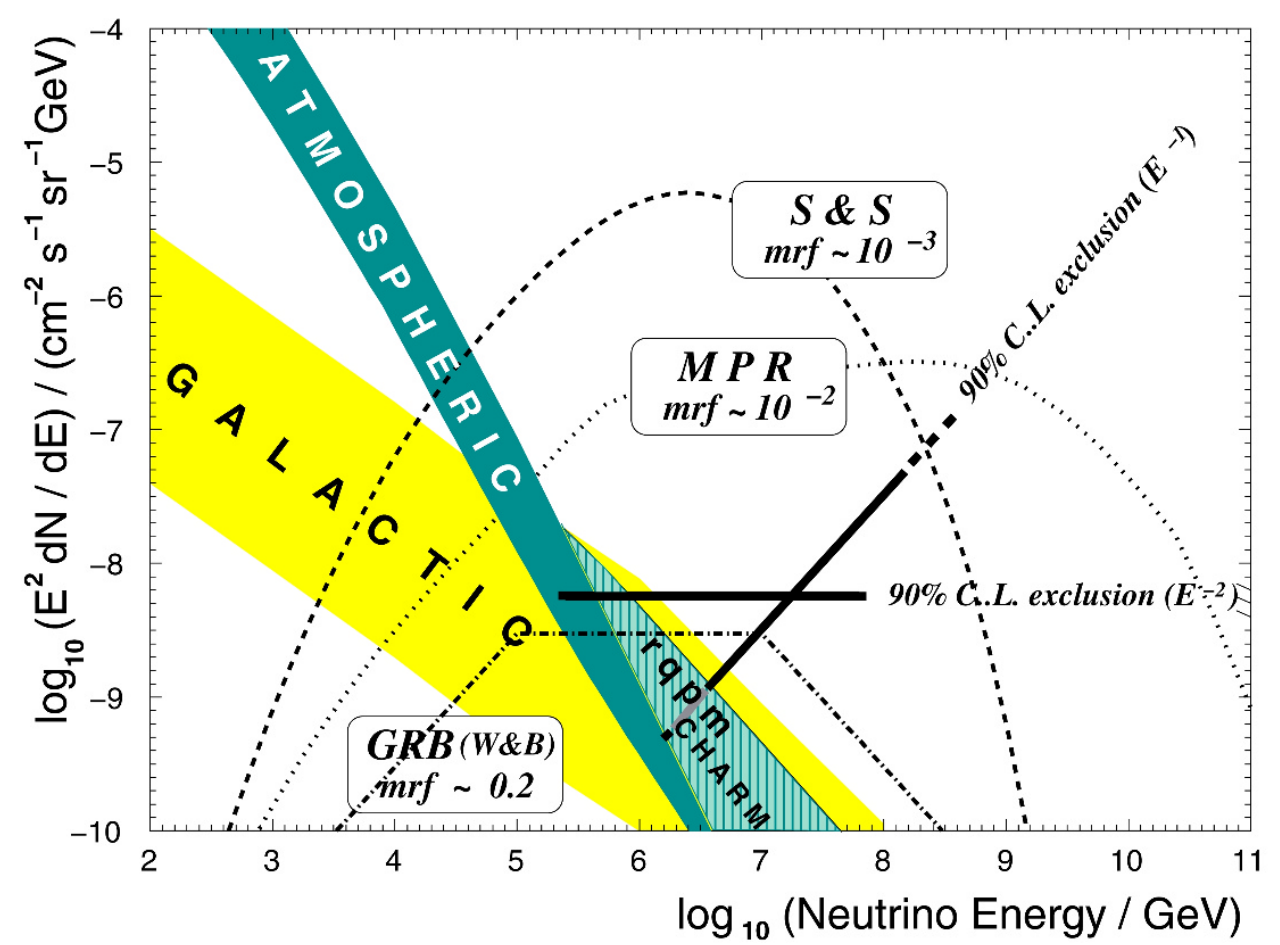

Fig. 1. Different contributions to the total astrophysical neutrino flux (Ahrens et al., 2004).

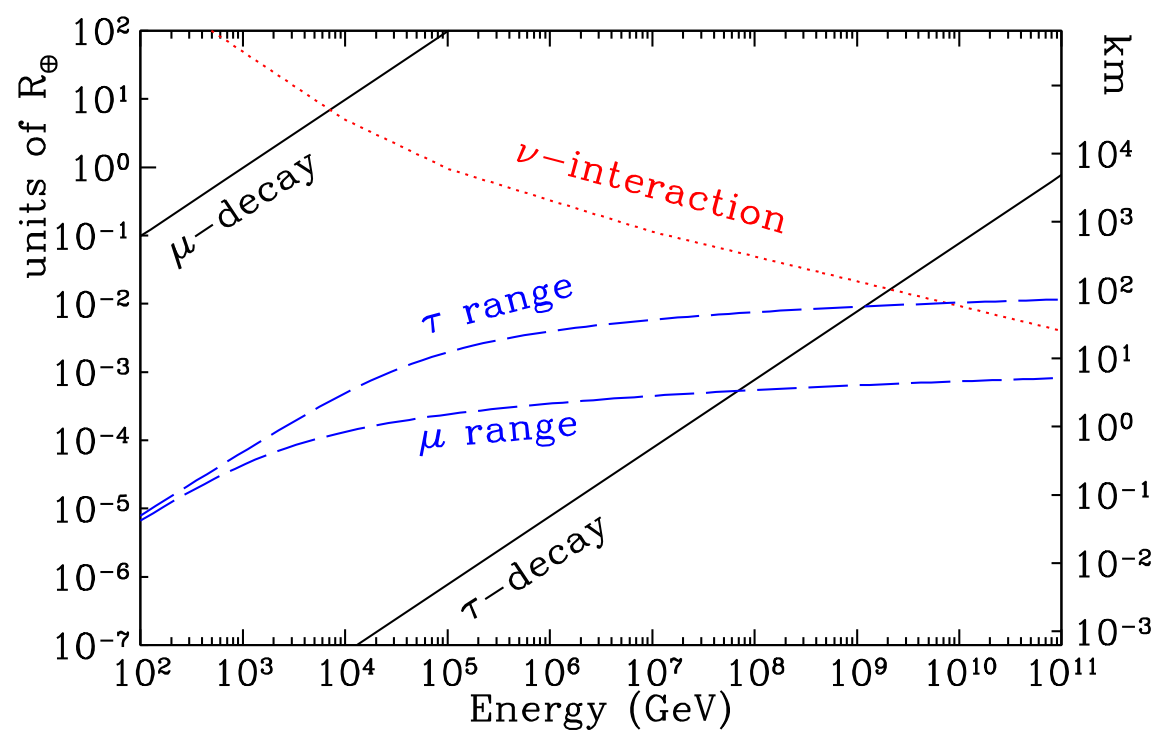

Fig. 2. Neutrino and charged leptons interaction/decay lengths (Beacom et al., 2002) vs energy.

where $d \Phi / d \Omega$ (expressed in $\mathrm{s}^{-1} \mathrm{~m}^{-2} \mathrm{sr}^{-1} \mathrm{GeV}^{-1}$ ) represents the solid angle distribution of neutrino flux, $A_{\text {eff }} \approx$ $\rho \sigma N_{\mathrm{A}} V_{\text {eff }}=V_{\text {eff }} / \lambda$ stands for the effective area, and $\Omega_{\mathrm{d}}$ is the integration solid angle. Since the effective detector volume is $V_{\text {eff }}=A_{\mathrm{p}} l$, where $A_{\mathrm{p}}$ is the detector area projected against the neutrino direction and $l$ is the portion of the neutrino path to which the detector is sensitive, the events rate can be rewritten as

$$
\Gamma\left(E_{v}\right)=\Omega_{\mathrm{d}} \frac{d \Phi}{d \Omega} \frac{A_{\mathrm{p}} l}{\lambda} .
$$

An interesting example of how sensitive is the neutrino interaction probability to matter distribution is provided by
Ultra High Energy $v_{\tau}$ 's which can be indirectly detected via their production of the charged tau's. Such leptons, once produced by charged current weak interaction, can emerge from surface if they have crossed few tens of km's in rock only (Earth-skimming events), see Fig. 2. The detection of UHE Earth-skimming neutrinos can be performed both from giant array detectors like Pierre Auger Observatory (PAO) or from $\mathrm{km}^{3} \mathrm{NT}$. Concerning PAO performances, they have been studied in a series of papers (Miele et al., 2006; Góra et al., 2007). As shown in Fig. 3, by starting from a Digital Elevation Model (DME) of the area nearby PAO one can compute, track by track hitting the experimental set up, the amount of rock really crossed. In Ref (Góra 


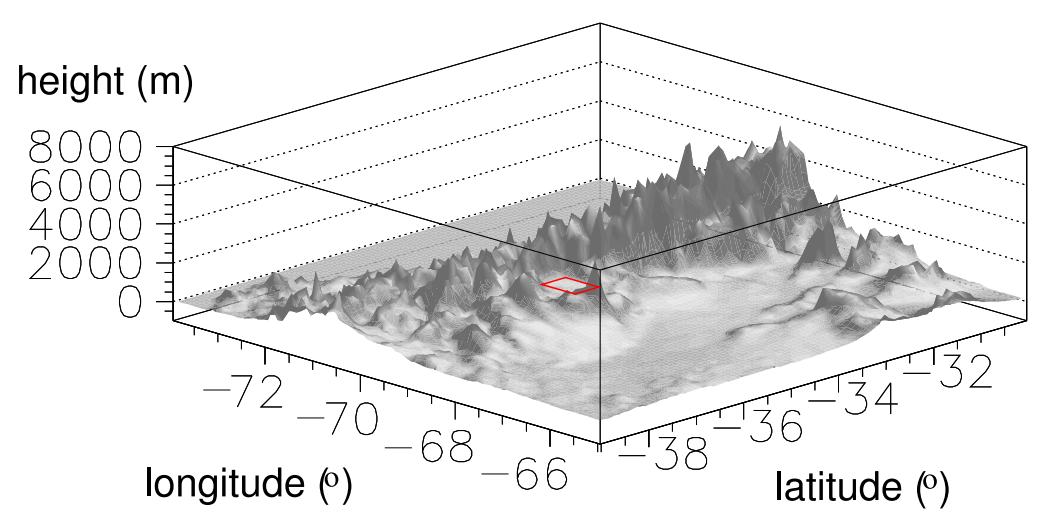

Fig. 3. A 3D map in longitude and latitude of the area around PAO with the elevation expressed in meters. The PAO position and surface, approximated to a rectangle, is indicated in red (Miele et al., 2006).

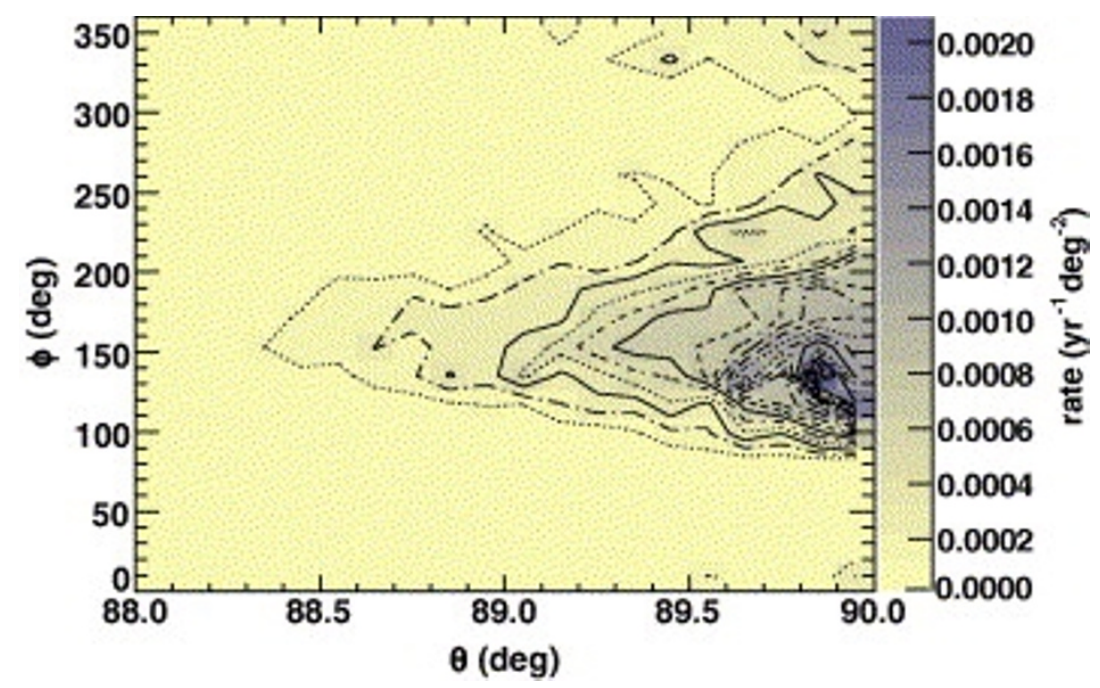

Fig. 4. Angular distribution of almost Horizontal event rate at Surface Detector of PAO. (Reprinted from Astropart. Phys., 26, Góra et al., A MC approach to simulate up- and down-going neutrino showers including local topographic conditions, 402, Copyright 2007, with permission from Elsevier.)

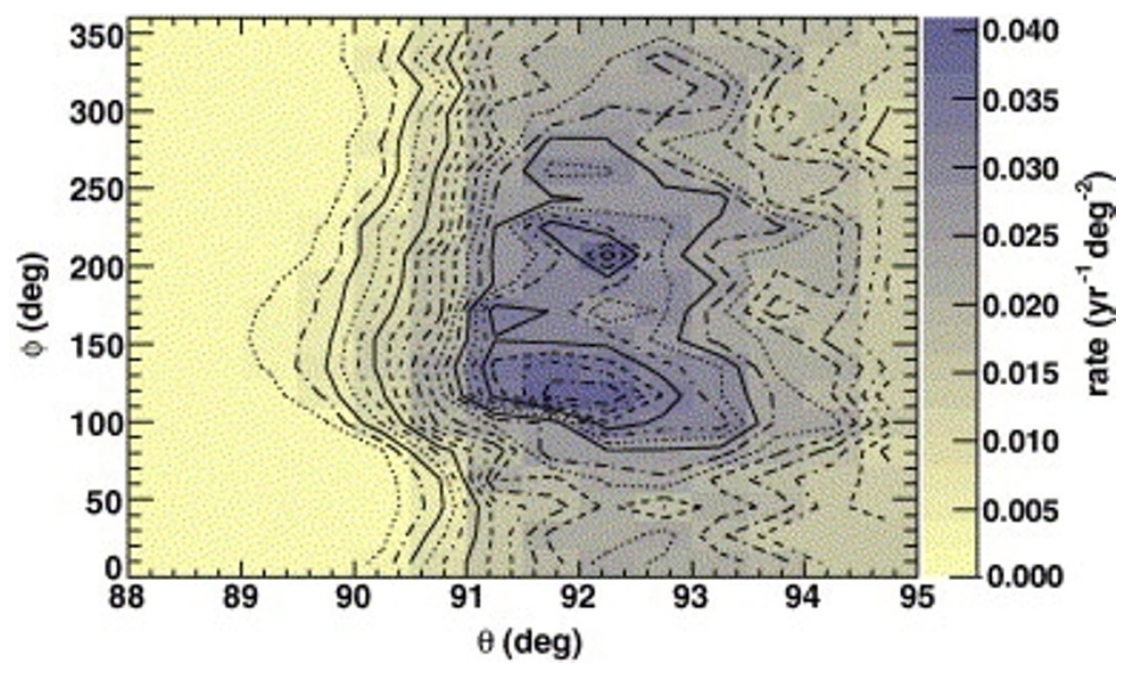

Fig. 5. Angular distribution of up-going Earth-skimming event rate at Surface Detector of PAO. (Reprinted from Astropart. Phys., 26, Góra et al., A MC approach to simulate up- and down-going neutrino showers including local topographic conditions, 402, Copyright 2007, with permission from Elsevier.) 


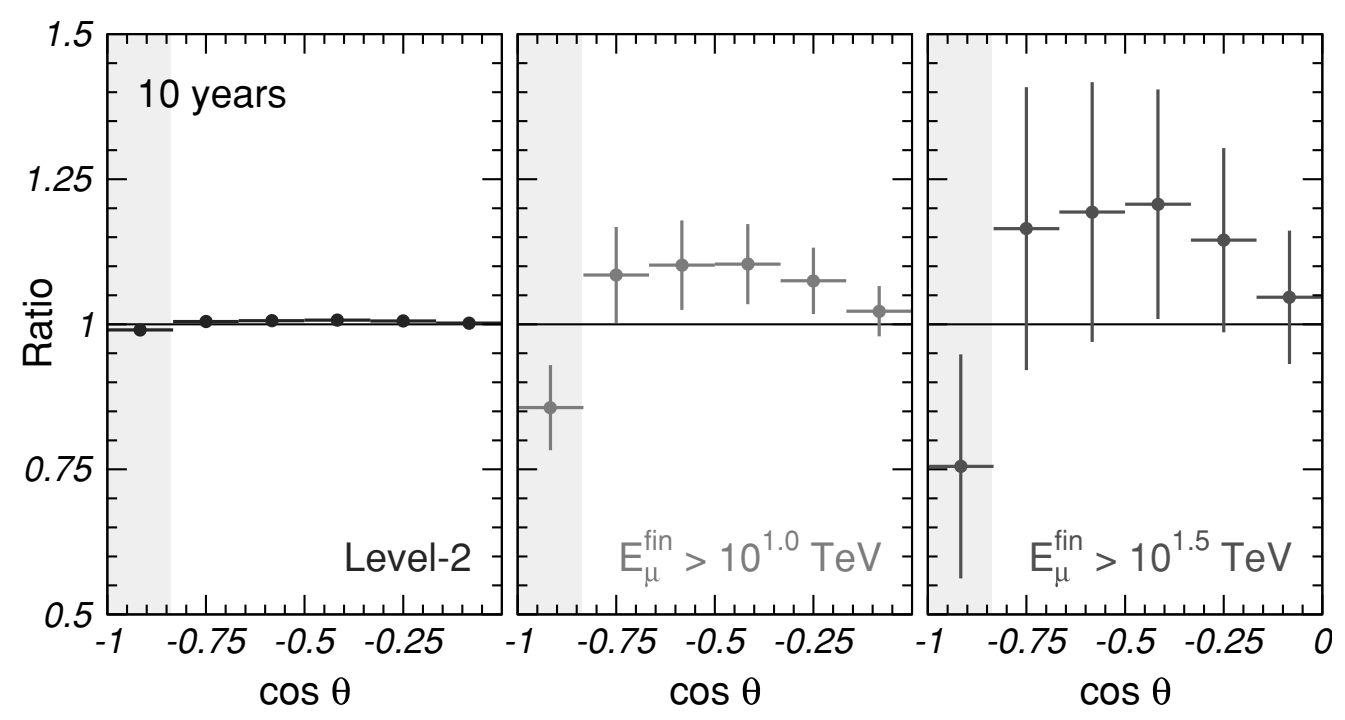

Fig. 6. Ratio of zenith angle distribution of expected events for the PREM over the expectations with an homogeneous Earth matter distribution for different values of the energy threshold of the events. The error bars in the figure show the expected statistical error in 10 years of IceCube. (González-García et al., 2008). Note that Level-2 denotes a background rejection quality cut defined in Ahrens et al. (2004) and Achterberg et al. (2006).

et al., 2007) the distribution of arrival directions of quasi horizontal and up-going Earth-skimming events have been computed, respectively. As one can see from Figs. 4 and 5 the effect of the matter distribution is very tiny, but not vanishing, and would require many years to be detected at PAO Surface Detector. A situation even worse is expected at the PAO Fluorescence Detector where a duty cycle of almost $10 \%$ is at work. Nevertheless, an enhancement in the event rate, due to matter effect, is present, and it can be interpreted as a naive neutrino radiography of superficial geological structures. The prediction for the event rate are function of the unknown UHE neutrino flux which, in Góra et al. (2007), has been assumed as the conservative Waxman-Bahcall limit. Of course a more optimistic flux could increase very much the number of events. In this concern the matter effect can also be used as a way to increase the detection performances of the apparatus for UHE neutrinos which are a relevant component of the extragalactic radiation and carry information about the acceleration mechanisms in extreme astrophysical environments. This topic was extensively studied in Cuoco et al. (2007) and Borriello et al. (2008) where the effect of matter distributed nearby an under-sea NT is evaluated for the three proposed Mediterranean sites. More recently the possibility to use the tail of atmospheric neutrinos to make a scan of the internal part of the Earth, namely the core/mantle transition zone, has been envisaged in Ref. (González-García et al., 2008). According to the authors a ten years running time of IceCube would allow to disentangle between a really homogeneous radial density profile and the already well-known Preliminary Reference Earth Model (PREM) (Dziewonski and Anderson, 1981). In particular in Fig. 6 the ratio between the expected event rates for the PREM and the homogeneous model, for different energy thresholds, are reported. As it is clear from the plots, increasing the energy threshold, namely by using more energetic neutrinos, the ratio becomes more and more sensitive to the proper radial density profile. Unfortunately, by using more energetic particles one loses statistics since their flux decreases with a power law, thus it is crucial to find a good compromise between sensitivity to the relevant physical quantity (PREM) and proper statistics required in order to make a statement with a good level of confidence.

\section{Conclusions}

Geoparticle physics is a fast growing new discipline which aims to export the large amount of know-how produced in a mature sector like elementary particle physics to geophysics. This idea has already good example of application especially in the use of muons. However, neutrinos either produced by the decay of radioactive nuclei (low energy $v$-typically denoted as geo-neutrinos) or more energetic ones, like the atmospheric- $v$ can give new and fascinating insights of the very deep interior of our planet. Unfortunately, due to the very elusive nature of these particles, in order to collect proper statistics one needs enormous detectors of at least $\mathrm{km}^{3}$ scale, which however are planned and even under-construction in some cases. Hence, in the near future by using these giant apparatus we will be able to see in practice the potentiality of this new technique.

Acknowledgments. G.M. acknowledges supports by the Spanish MICINN (grants SAB2006-0171 and FPA2005-01269) and by INFN I.S. Fa51 and PRIN 2006 Fisica Astroparticellare: Neutrini ed Universo Primordiale of Italian MIUR.

\section{References}

Achterberg, A. et al. [IceCube Collaboration], First year performance of the IceCube neutrino telescope, Astropart. Phys., 26, 155, 2006.

Aggouras, G. et al. [NESTOR Collaboration], Recent results from NESTOR, Nucl. Instrum. Meth. A, 567, 452, 2006.

Ahrens, J. et al. [AMANDA Collaboration], Observation of high-energy atmospheric neutrinos with the Antarctic Muon and Neutrino Detector Array, Phys. Rev. D, 66, 012005, 2002.

Ahrens, J. et al. [IceCube Collaboration], Sensitivity of the IceCube detector to astrophysical sources of high energy muon neutrinos, Astropart. 
Phys., 20, 507, 2004.

Alvarez, L. W., J. A. Anderson, F. El Bedwei, J. Burkhard, A. Fakhry, A. Girgis, A. Goneid, F. Hassan, D. Iverson, G. Lynch, Z. Miligy, A. H. Moussa, M. Sharkawi, and L. Yazolino, Search for hidden chambers in the pyramids, Science, 167, 832, 1970.

Balkanov, V. A. et al., Registration of atmospheric neutrinos with the BAIKAL neutrino telescope NT-96, Astropart. Phys., 12, 75, 1999.

Beacom, J. F., P. Crotty, and E. W. Kolb, Enhanced signal of astrophysical tau neutrinos propagating through Earth, Phys. Rev. D, 66, 021302, 2002.

Borriello, E., A. Cuoco, G. Mangano, G. Miele, S. Pastor, O. Pisanti, and P. D. Serpico, Disentangling neutrino-nucleon cross section and high energy neutrino flux with a km3 neutrino telescope, Phys. Rev. D, 77, 045019, 2008

Cuoco, A., G. Mangano, G. Miele, S. Pastor, O. Pisanti, and P. D. Serpico, Ultrahigh energy neutrinos in the Mediterranean: Detecting v(tau) and $\mathrm{v}(\mathrm{mu})$ with a km3 Telescope, JCAP, 0702, 007, 2007.

Dziewonski, A. M. and D. L. Anderson, Preliminary Reference Earth Model, Phys. Earth Planet. Inter., 25(4), 297-356, 1981.

George, E. P., Cosmic rays measure overburden of tunnel, Commonwealth Engineer, July 1, 455, 1955.
González-García, M. C., F. Halzen, M. Maltoni, and H. K. M. Tanaka, Radiography of earth's core and mantle with atmospheric neutrinos, Phys. Rev. Lett., 100, 061802, 2008.

Góra, D., M. Roth, and A. Tamburro, A MC approach to simulate up- and down-going neutrino showers including local topographic conditions, Astropart. Phys., 26, 402, 2007.

Katz, U. F., KM3NeT: Towards a km**3 Mediterranean neutrino telescope, Nucl. Instrum. Meth. A, 567, 457, 2006.

Miele, G., S. Pastor, and O. Pisanti, The aperture for UHE tau neutrinos of the Auger fluorescence detector using a digital elevation map, Phys. Lett. B, 634, 137, 2006.

Migneco, E. et al. [NEMO Collaboration], Recent achievements of the NEMO project, Nucl. Instrum. Meth. A, 588, 111, 2008.

Spurio, M. [ANTARES Collaboration], Status report (2006) of the ANTARES project, 2006.

E. Borriello, G. De Lellis, G. Mangano, A. Marotta, G. Miele (e-mail: miele@na.infn.it), P. Migliozzi, C. Moura, S. Pastor, O. Pisanti, P. Strolin, and A. Zollo 